\title{
ALIMENTAÇÃO COMPLEMENTAR DO LACTENTE: SUBSÍDIOS PARA A CONSULTA DE ENFERMAGEM EM PUERICULTURA*
}

\author{
Carmina Silva Santos ${ }^{1}$, Maria Wanderleya de Lavor Coriolano ${ }^{2}$, Gabriela Cunha Schechtman Sette ${ }^{3}$, Luciane Soares
}

de Lima ${ }^{4}$

RESUMO: O presente artigo teve por objetivo investigar os fatores que influenciam, positiva ou negativamente, a alimentação complementar do lactente, a partir de publicações científicas no período de 1990-2009. O método foi a revisão integrativa da literatura nas bases de dados Medline, BDENF e Lilacs utilizando os descritores aleitamento materno, desmame e nutrição do lactente. Os resultados apontam que o padrão alimentar é fator de grande importância para a manutenção da saúde da criança, sendo delineado por um conjunto de fatores que provêm do contexto familiar, condições socioeconômicas e culturais e nível de escolaridade da mãe/cuidador. Propõe-se, na consulta de enfermagem em puericultura e a construção compartilhada do cardápio infantil entre o enfermeiro e o cuidador com vistas a oferecer à criança uma educação alimentar que previna doenças na vida adulta.

PALAVRAS-CHAVE: Aleitamento materno; Desmame; Nutrição do lactente; Enfermagem.

\section{SUPPLEMENTARY FEEDING OF INFANTS: ALLOWANCES FOR CONSULTATION IN NURSING CHILDCARE}

\begin{abstract}
This paper aimed to investigate the factors that influence, positively or negatively, complementary feeding of infants, from scientific publications in the period from 1990 to 2009. The method was the integrative literature review in the databases Medline, LILACS and BDENF using the keywords breastfeeding, weaning and infant nutrition. The results show that the eating pattern is a very important factor for maintaining the health of children, and outlined a set of factors that come from family background, socioeconomic and cultural and educational level of mother / caretaker. A shared construction of a children's menu is proposed in the nursing consultation in child care, and between nurse and caregiver in order to offer children an education that will prevent food illnesses in adulthood.
\end{abstract}

KEYWORDS: Breast feeding; Weaning; Infant nutrition; Nursing.

\section{ALIMENTACIÓN COMPLEMENTAR DEL LACTENTE: SUBSIDIOS PARA LA CONSULTA DE ENFERMERÍA EN PUERICULTURA}

\begin{abstract}
RESUMEN: El presente artículo tuvo por objetivo investigar los factores que influenccían, positiva o negativamente, la alimentación complementar del lactente, con base en publicaciones científicas en periodo de 1990 a 2009 . El método fue la revisión integrativa de la literatura en las bases de datos Medline, BDENF y Lilacs, utilizando los descriptores amamantamiento materno, desmamar y nutrición del lactente. Los resultados apuntan que el patrón alimentar es factor de grande importancia para la manutención de la salud del niño, siendo delineado por un conjunto de factores que provienen del contexto familiar, condiciones socioeconómicas y culturales y nivel de escolaridad de la madre/cuidador. Se propone la consulta de enfermería en puericultura y la construcción compartillada del menú infantil entre el enfermero y el cuidador a fin de ofrecer al niño una educación alimentar que prevenga enfermedades en la vida adulta.
\end{abstract}

PALABRAS CLAVE: Amamantamiento materno; Desmamar; Nutrición del lactante; Enfermería.

\footnotetext{
*Extraído da Dissertação de Mestrado "Principais problemas encontrados pelas mães de crianças entre cinco e oito meses relativos à transição alimentar atendidas em um serviço de puericultura do Recife", apresentada ao Programa de Pós-Graduação em Saúde da Criança e do Adolescente, Universidade Federal de Pernambuco-UFPE.

${ }^{1}$ Enfermeira Assistencial do setor de Puericultura e Vacina do Instituto de Medicina Integral Professor Fernando Figueira-IMIP. Mestre em Saúde da Criança e do Adolescente. Coordenadora de Tutores de Enfermagem na Escola Pernambucana de Saúde-FBV/IMIP. ${ }^{2}$ Enfermeira. Mestranda em Saúde da Criança e do Adolescente pelo Programa de Pós-Graduação da UFPE. Bolsista CNPq. ${ }^{3}$ Enfermeira. Professora Substituta do Departamento de Enfermagem da UFPE. Mestranda pelo Programa de Pós-Graduação em Saúde da Criança e do Adolescente da UFPE. Bolsista CAPES.

${ }^{4}$ Enfermeira. Doutora em Ciências Pneumológicas. Professora do Departamento de Enfermagem da UFPE. Professora do Programa de Pós-Graduação da UFPE.
}

Autor correspondente:

Luciane Soares de Lima

Universidade Federal de Pernambuco

Rua do Futuro, 77/1101 - 520-50010 - Recife-PE, Brasil

Recebido: 24/12/09

Email: luciane.lima@globo.com

Aprovado: 26/06/10

Cogitare Enferm. 2010 Jul/Set; 15(3):536-41 


\section{INTRODUÇÃO}

A realização do estudo representa uma resposta a questionamentos no exercício da consulta de enfermagem, ao verificar a frequente ocorrência de não-adequação das mães às orientações do Ministério da Saúde (MS) com relação à introdução da alimentação complementar de seus filhos. Isto fez surgir a pergunta: Por que as mães, sendo orientadas em um Serviço de Puericultura, não seguem as orientações no que se refere à alimentação complementar do lactente?

Imaginou-se, também, que enfermeiros que realizam puericultura em outros serviços poderiam ter os mesmos questionamentos. Haja vista o advento dos Programas de Saúde da Família, distribuídos, em 2004, em $84 \%$ dos municípios brasileiros, com efetiva participação do enfermeiro nas equipes multidisciplinares, também motivou as autoras a tentar esclarecer estes questionamentos, através deste estudo ${ }^{(1-2)}$.

O processo alimentar infantil é mediado por diversos fatores intervenientes, tais como o contexto familiar, condições socioeconômicas e culturais e nível de escolaridade da mãe/cuidador sobre o hábito de alimentar a criança. Estes fatores constituem o objeto do presente estudo, sob a forma de revisão bibliográfica, do tipo revisão integrativa, e cujo objetivo foi investigar os fatores que influenciam positiva ou negativamente na alimentação complementar do lactente, a partir de publicações científicas no período de 1990-2009.

Os subsídios coletados poderão contribuir para o maior conhecimento do tema e serem utilizados nas práticas inerentes ao exercício da consulta de puericultura pelos enfermeiros, com maior compreensão dos fatores envolvidos na alimentação complementar do lactente.

\section{METODOLOGIA}

Trata-se de uma revisão integrativa da literatura, definida como revisão de literatura, que inclui a análise de pesquisas relevantes que dão suporte para a tomada de decisão e a melhoria da prática clínica ${ }^{(3)}$. Foi baseada em material indexado nas bases de dados Medical Literature Analysis and Retrieval System On-Line (Medline), Base de Dados de Enfermagem (BDENF), Literatura Latino-Americana e do Caribe em Ciências da Saúde (LILACS) e informações referentes a consensos do Ministério da Saúde e Organização Mundial da Saúde, livros, dissertações e monografias publicados no período de 1990-2009.

Foram delimitados como descritores: aleitamento materno, nutrição do lactente e desmame. Cruzando-se os descritores aleitamento materno e desmame foram encontradas no LILACS 237 publicações, MEDLINE 471 publicações e BDENF 0 artigos; usando os descritores aleitamento materno e nutrição do lactente, 226 publicações no LILACS, 0 no MEDLINE e 0 na BDENF. Quando foram cruzados os descritores desmame e nutrição do lactente, foram encontradas 43 publicações no Lilacs, 0 no MEDLINE e 0 no BDENF. Ao serem cruzados os três descritores, foram encontrados no LILACS 27 publicações, 0 no MEDLINE e 0 no BDENF. A revisão foi direcionada na abordagem da influência negativa ou positiva de fatores intervenientes, tais como o contexto familiar, condições socioeconômicas e culturais, e nível de escolaridade sobre o hábito alimentar da criança.

Após leitura cuidadosa dos artigos e resumos, foram selecionados para responder o objetivo da revisão oito artigos que respondiam à questão de pesquisa, somados a dez publicações, incluindo dissertação(1), monografia $^{(1)} \mathrm{e}$ livros da área técnica ${ }^{(3)}$. Das demais publicações mencionadas, quatro eram consensos do Ministério da Saúde e Organização Mundial da Saúde no que se refere à alimentação complementar, e quatro livros da área de nutrição.

Consideraram-se como artigos elegíveis: originais, revisão e reflexão, sendo que após a leitura dos resumos e textos completos relacionados ao objeto de estudo, incluiu-se oito artigos de pesquisa. A análise das publicações permitiu a identificação de quatro temáticas: fatores intervenientes na alimentação complementar; fatores relacionados à mãe/cuidador; tipos de alimentos, preparo e utensílios utilizados para a oferta; repercussões da inadequação da alimentação complementar sobre a saúde da criança.

\section{RESULTADOS E DISCUSSÃO}

O conceito de alimentação complementar adotado foi do Ministério da Saúde, o qual define que a partir dos seis meses de idade, a alimentação, conforme o nome sugere, tem a função de complementar a energia e micronutrientes necessários para o crescimento saudável e pleno desenvolvimento das crianças. As situações mais comuns relacionadas à alimentação complementar oferecida de forma inadequada são: anemia, excesso de peso e desnutrição ${ }^{(4)}$. A partir da leitura dos artigos selecionados, além das 
publicações técnicas, foram delimitadas as temáticas referentes à alimentação complementar do lactente apresentadas a seguir.

Fatores intervenientes na alimentação complementar

Inicialmente, deve-se considerar que o padrão alimentar da criança envolve participação a efetiva dos pais como educadores nutricionais, quase sempre determinante no comportamento alimentar da criança ${ }^{(5)}$.

Este é direcionado, primariamente, pela família da qual é dependente e, secundariamente, por outras interações psicossociais e culturais da criança ${ }^{(6)}$. Gradativamente, a criança recebe a alimentação dos pais que têm a responsabilidade de oferecer um cardápio variado para que ela conheça os diversos sabores, desenvolvendo e exercitando seu paladar ${ }^{(1)}$.

Pode-se afirmar que a família é responsável pela cultura alimentar, as escolhas alimentares, a quantidade e qualidade dos alimentos, o tempo e o intervalo para comer, enfim, ela é responsável pelo estabelecimento de regras e normas no processo de alimentação da criança ${ }^{(6)}$.

O contexto social é de grande importância na alimentação, uma vez que afeta a experiência alimentar, influencia os padrões de alimentação e o desenvolvimento socioemocional da criança. A qualidade da relação pais-filhos, durante os primeiros anos de vida da criança, está relacionada à alimentação, iniciando-se com a amamentação ${ }^{(5)}$.

Por este motivo, a família é responsável pela transmissão da cultura alimentar. Na sua socialização a criança aprende sobre a sensação de fome e saciedade e desenvolve a percepção para os sabores e as suas preferências, iniciando a formação do seu comportamento alimentar ${ }^{(7)}$. O padrão alimentar da criança é diretamente influenciado pelas condições socioeconômicas, com ênfase na distribuição dos alimentos na família (quem come primeiro, se em prato separado), bem como no poder de decisão do mantenedor da família sobre quais alimentos serão consumidos $^{(8)}$.

É preciso também considerar as percepções e crenças que exercem influência sobre a família, pois o consumo alimentar de determinados grupos está vinculado aos diversos valores e sentimentos que se relacionam com aspectos da cultura, transpondo o ato biológico de comer e preenchendo funções simbólicas na vida dos indivíduos. Em consequência disto, geram-se diversas restrições e exclusões alimentares, algumas antigas e descritas desde o início da história da alimentação $^{(9)}$.

Nos dias de hoje, como exemplos, podem ser citados alguns tabus comuns na região Nordeste, em relação ao feijão ("é pesado"), pinha ("é reimosa"), manga ("se oferecida com leite. mata"), batata doce, jerimum e folhas verdes de consumo usual ("fracol comida de coelho"), banana no horário da tarde ("é pesada"), dentre outros ${ }^{(9)}$. Para uma orientação alimentar segura, é necessário conhecer e saber lidar com as percepções e crenças da família acerca da saúde e nutrição da criança. Isto é um importante aspecto a ser considerado e, sempre que possível, estas percepções e crenças devem ser consideradas e não combatidas durante o processo de educação em saúde ${ }^{(6)}$.

Neste âmbito, o contexto familiar assume a sua importância na inserção da alimentação complementar, tendo em vista as crenças e saberes que são adquiridos historicamente, a interação com os profissionais que assistem a criança e família, a interação e afetividade com a criança, além de fatores econômicos que influenciam na aquisição de alimentos para suprimento da família.

Todos estes aspectos necessitam ser considerados durante a consulta de enfermagem em puericultura, objetivando garantir um aporte nutricional para as crianças nesta etapa do seu ciclo de vida.

\section{Fatores relacionados à mãe/cuidador}

Em relação ao papel assumido pelo cuidador principal, são destacados alguns fatores que influenciam a prática da alimentação complementar, dos quais podem ser citados: o conhecimento das mães/ cuidadores sobre alimentação infantil; as condições de saúde do lactente e da mãe; valores culturais, crenças, tabus, estilo de vida da mãe ou de quem cuida da criança; recursos disponíveis para o preparo dos alimentos complementares em condições adequadas, inclusive de higiene; ajuda para cuidar da criança e dos afazeres domésticos; poder aquisitivo das famílias; e recomendações familiares, dos amigos e dos profissionais de saúde ${ }^{(8,10-11)}$.

Há ainda um aspecto importante associado a todos estes fatores, que pode dificultar a introdução dos alimentos complementares: as crianças que não querem comer outros alimentos, por preferir o leite materno. Alguns lactentes procuram constantemente a mama, mais por quer a presença, o carinho, o colo da mãe do que por estar com fome. As mamadas 
frequentes, nestas circunstâncias, inibem a fome e fazem com que a criança não aceite os alimentos complementares $^{(10)}$.

Em estudo sobre práticas alimentares para crianças nos primeiros meses de vida, afirma-se que as chances de introdução oportuna de alimentos sólidos crescem com o aumento da idade materna e o nível de escolaridade ${ }^{(12)}$.

Em outro estudo sobre aleitamento materno e prática de alimentação complementar, observou-se que a adesão às recomendações sobre alimentação da criança está associada à educação materna. Foi também demonstrado, em estudo que propõe estratégias para aumentar os conhecimentos das mães em aleitamento materno, que a escolaridade materna interfere significativamente no conhecimento sobre amamentação $^{(12)}$.

O cuidador principal, como explicitado, assume um papel de maior destaque por envolverse na aquisição de informações sobre alimentação complementar, preparo dos alimentos, e oferecimento ao lactente, sendo indispensável para o enfermeiro manter uma relação de confiança e empatia com este sujeito identificando dificuldades que comprometam a introdução e manutenção adequada dos alimentos complementares.

\section{Tipos de alimentos, preparo e utensílios utilizados para a oferta}

Em um encontro de profissionais de saúde do Nordeste ${ }^{(6)}$, foi verificado que, nesta região, de forma geral, o padrão alimentar complementar é inadequado. Isto acontece porque os alimentos ministrados às crianças são de baixo teor energético, devido à diluição inadequada (leite de vaca em pó e in natura) e ao uso de preparações predominantemente líquidas e diluídas (caldos de arroz, feijão, vegetais ou legumes). A utilização de frutas e vegetais é limitada. As dietas têm pouca variabilidade, são pobres em vitaminas e sais minerais, não refletindo as opções locais de variação alimentar.

A dieta monótona acarreta deficiência de nutrientes específicos, contribuindo para a anorexia crônica. Estas dietas, muitas vezes, são oferecidas sob forma líquida e, comumente, em mamadeira. Sabe-se que mesmo às crianças que ainda se amamenta são administradas mamadeiras com sucos, chás e água ${ }^{(6-7)}$.

Esta prática constitui importante fonte de contaminação dos alimentos dos bebês, sabendo-se que as mamadeiras são difíceis de higienizar, conforme afirmam as próprias mães. Isto reflete a deficiência nas práticas de higiene, que podem ocorrer durante o manuseio, o preparo, a administração e o armazenamento dos alimentos oferecidos às crianças. A higienização inadequada durante este processo repercute no aumento da mortalidade infantil por doenças diarreicas $^{(1)}$.

O papel dos alimentos complementares como uma das principais vias de transmissão de doenças diarreicas em crianças pequenas foi comprovado em estudo realizado no Peru, no qual foi demonstrado, do ponto de vista bacteriológico, a contaminação dos alimentos ${ }^{(8)}$. A influência negativa de práticas de higiene por mães de crianças menores de um ano, no que concerne à lavagem das mãos antes do preparo dos alimentos, higiene dos utensílios, bem como estocagem dos alimentos preparados, foi um resultado encontrado em outro estudo ${ }^{(9)}$.

Em Campinas (SP), verificou-se que o aleitamento materno exclusivo estava sendo interrompido aos 90 dias, momento no qual $50 \%$ das crianças não recebiam esse leite. Observou-se, ainda, que a introdução de alimentos complementares não estava dentro do preconizado pelo Ministério da Saúde, com oferecimento precoce principalmente de guloseimas e de outros alimentos não adequados à dieta infantil ${ }^{(14)}$.

Corroborando os achados explicitados anteriormente, em Botucatu (SP), evidenciou-se que a inserção da alimentação complementar ocorria precoce e inadequadamente, caracterizando a interrupção do aleitamento materno nos primeiros meses de vida, tendo como alimento inicial o chá, com a maior frequência de consumo em menores de quatro meses ${ }^{(14)}$.

\section{Repercussões da inadequação da alimentação complementar sobre a saúde da criança}

A saúde de uma população está intimamente relacionada com sua alimentação. A formação de hábitos alimentares saudáveis deve ser iniciada precocemente, no primeiro ano de vida ${ }^{(15)}$.

As doenças crônicas não transmissíveis são um reflexo dos hábitos alimentares e do estilo de vida e vêm crescendo, nos países em desenvolvimento, acarretando uma constante preocupação com os excessos na alimentação, muitas vezes iniciados nos primeiros anos de vida. No Brasil, observa-se um aumento frequente de obesidade na população infantil, aparentemente um paradoxo, considerando 
que, até pouco tempo, a desnutrição era a maior causa de preocupação, embora o excesso do consumo de carboidratos em detrimento de proteínas e outros micronutrientes seja uma preocupação atual nos estratos sociais menos favorecidos ${ }^{(16)}$.

$\mathrm{O}$ oferecimento de uma dieta variada possibilita um melhor fornecimento de micronutrientes, como ferro, zinco e vitamina A. A deficiência de ferro, tendo como consequência a anemia, é considerada a doença mais prevalente em todo o mundo, e chega a ser responsável por $95 \%$ desta patologia. Ela ocorre principalmente em crianças com idade inferior a 4 anos e em países em desenvolvimento ${ }^{(15)}$.

Na primeira infância, o problema se agrava em decorrência de erros alimentares, principalmente no período de desmame, quando frequentemente o leite materno é substituído por alimentos pobres em ferro, como o leite artificial. Nestes alimentos substitutos do leite materno, a biodisponibilidade de ferro é muito baixa e, como se sabe, as mães frequentemente substituem uma refeição pela mamadeira ${ }^{(16)}$. Outro problema comum durante a inserção de alimentos complementares é o ganho ponderal insuficiente da criança, o qual pode ser mensurado por meio do acompanhamento do crescimento, utilizando as curvas de referência padronizadas pelo Ministério da Saúde.

Assim, é evidente que práticas alimentares simples e factíveis, em especial para crianças pequenas, podem minimizar os efeitos da iniquidade social que atinge o país. A divulgação e implantação de práticas alimentares adequadas, embasadas cientificamente, podem reduzir os problemas alimentares da população, sobretudo nos segmentos sociais mais carentes.

\section{CONSIDERAÇÕES FINAIS}

A atuação do enfermeiro na abordagem alimentar em puericultura constitui-se numa atividade desafiadora, tendo em vista os múltiplos fatores envolvidos, tais como: utensílios utilizados no preparo e oferecimento dos alimentos, tipos de alimentos, condições socioeconômicas das famílias, hábitos culturais, aspectos emocionais relacionados ao cuidador e às crianças, condições de saúde do lactente, fase do desenvolvimento neuropsicomotor, estado nutricional e educação dos cuidadores.

Diante da possibilidade de investigações e intervenções durante a consulta de enfermagem, faz-se necessária a utilização de ferramentas de edu- cação em saúde que permitam a construção de um cardápio compatível com as necessidades nutricionais do lactente, considerando as condições e saberes dos cuidadores/famílias. Nesta perspectiva, propõe-se uma prática de construção compartilhada do cardápio infantil, com vistas a oferecer à criança uma educação alimentar que previna doenças na vida adulta.

\section{REFERÊNCIAS}

1. Ministério da Saúde (BR). Guia alimentar para crianças menores de 2 anos. [Internet] Brasília; 2002 [acesso em 30 nov 2009]. Disponível: http://tiny.cc/bjj93

2. Escorel S, Giovanella L, Mendonça MHM, Senna MCM. O Programa de Saúde da Família e a construção de um novo modelo para a atenção básica no Brasil. Rev Panam Salud Publica. 2007;21(2):164-76.

3. Mendes KDS, Silveira RCCP, Galvão CM. Revisão integrativa: método de pesquisa para a incorporação de evidências na saúde e na enfermagem. Texto Contexto Enferm 2008;17(4): 758-64.

4. Ministério da Saúde (BR). Saúde da criança: nutrição infantil - aleitamento materno e alimentação complementar. Brasília; 2009.

5. Vieira MLF, Pinto JLC, Barros AAF. Amamentação e a alimentação complementar de filhos de mães adolescentes são diferentes dos de filhos de mães adultas? J Pediatr. 2003;79(4):317-24.

6. Monte CMG, Sá MLB. Guias alimentares para crianças menores de dois anos no Nordeste do Brasil: da teoria à prática; Brasília;1999.

7. Cascudo LC. História da alimentação no Brasil. Belo HorizontelSão Paulo: ItatiaialEdusp; 1983.

8. World Health Organization. The World Health Organization's infant-feeding recommendations. Bull World Health Organ. 1995;73:165-74.

9. Monte CMG. Normas de atenção à criança desnutrida nos diversos níveis dos serviços de saúde. Subsídio para discussão do grupo consultor de desnutrição infantil para o Ministério da Saúde. Fortaleza; 2000.

10. Giugliani ERJ, Victora CG. Normas alimentares para crianças brasileiras menores de dois anos: bases científicas. Brasília:OPAS\OMS; 1997.

11. Frota MA, Aderaldo NNS, Silveira VG, Rolim 
KMC, Martins MC. O reflexo da orientação na prática do aleitamento materno. Cogitare Enferm. 2008;13(3):403-9.

12. Lande B. Infant feeding practices and associated factors in the first six months of life: the norwegian infant nutrition survey. Acta Pediatr. 2003;92:152-61. duplicada

13. Bernardi JLD, Jordão RE, Barros Filho AA. Alimentação complementar de lactentes em uma cidade desenvolvida no contexto de um país em desenvolvimento. Rev Panam Salud Publica. 2009;26(5):405-11.

14. Parada CMGLM, Carvalhaes MABL, Jamas MT. Práticas de alimentação complementar em crianças no primeiro ano de vida. Rev Latino-Am Enfermagem [Internet]. 2007; 15(2) [acesso em 30 nov 2009]. Disponível: http://tiny.cc/pxcau

15. Monteiro CA, Szarfarc SC, Mondini L. Tendência secular da anemia na infância na cidade de São Paulo (1984-1996). Rev Saúde Publica. 2000; 6(supl):62-72. Rever abreviatura do periódico no IBICT

16. Osório MM. Fatores determinantes da anemia em crianças. J Pediatr. 2002;78(4):269-78. 